\title{
R1467H variant in the rho guanine nucleotide exchange factor 11 (ARHGEF11) is associated with impaired glucose tolerance and type 2 diabetes in German Caucasians
}

\author{
Yvonne Böttcher · Dorit Schleinitz · Anke Tönjes · Matthias Blüher · \\ Michael Stumvoll · Peter Kovacs
}

Received: 19 November 2007/Accepted: 6 January 2008/Published online: 31 January 2008

(C) The Japan Society of Human Genetics and Springer 2008

\begin{abstract}
The human rho guanine nucleotide exchange factor 11 (ARHGEF11) functions as an activator of rho GTPases and is supposed to influence insulin signalling. We investigated the effects of the previously reported $\mathrm{R} 1467 \mathrm{H}$ variant in individual's susceptibility to type 2 diabetes (T2D) and impaired glucose tolerance (IGT) as well as related traits in a German Caucasian cohort. Our study replicated associations of the $\mathrm{R} 1467 \mathrm{H}$ with increased risk of T2D or T2D/IGT, thus, implicating a potential role of ARHGEF11 in the aetiology of T2D and IGT.
\end{abstract}

Keywords Type 2 diabetes - Genetics .

Genetic association · Polymorphism - Candidate gene

Type 2 diabetes (T2D) is one of the most common human endocrine disorders. Its aetiology has a strong genetic background, which is still incompletely known. To search for genetic loci that contribute to T2D several genomic linkage scans have been completed worldwide (McCarthy 2003). One of the strongest evidence for linkage with T2D was centred on chromosome 1q21-q24 and has been widely

Y. Böttcher $(\varangle) \cdot$ A. Tönjes · M. Blüher · M. Stumvoll

Medical Department III, University of Leipzig,

Philipp-Rosenthal-Str. 27, 04103 Leipzig, Germany

e-mail: yvonne.boettcher@medizin.uni-leipzig.de

D. Schleinitz · P. Kovacs $(\bowtie)$

Interdisciplinary Center for Clinical Research IZKF,

University of Leipzig, Inselstrasse 22, 04103 Leipzig, Germany

e-mail: peter.kovacs@medizin.uni-leipzig.de

A. Tönjes

Coordination Center for Clinical Trials,

University of Leipzig, Leipzig, Germany replicated in populations with various ethnic background (McCarthy 2003). To identify the genetic variant(s) that gave rise to the linkage to T2D, positional candidate genes that map near 1q21-24 are being analyzed. One such candidate is the ARHGEF11 (rho guanine nucleotide exchange factor 11) gene. ARHGEF11 is an activator of rho GTPases and controls numerous cellular processes, including insulin signalling (Nevins and Thurmond 2005) and insulin secretion (Larsen et al. 2005). Therefore, ARHGEF11 is also a plausible physiologic candidate gene that might influence individual's susceptibility to insulin resistance and T2D.

Recently, R1467H variant (rs945508) in the ARHGEF11 was reported to be associated with increased risk for T2D and insulin resistance in Pima Indians of Arizona (Ma et al. 2007). Furthermore, genetic variation in the ARHGEF11 was shown to be associated with T2D and impaired glucose tolerance (IGT) also in the Old Order Amish (Fu et al. 2007). However, in contrast to the findings in Pima Indians the more common R1467 allele was identified as a risk variant for T2D/IGT in the Amish. This inconsistency in the effects of genetic variants may be due to different genetic backgrounds between the two ethnic groups.

Therefore, in the present study we attempted to replicate the effects of this variant in individual's susceptibility to T2D and IGT as well as its relation to metabolic parameters in German Caucasians.

A total of 685 unrelated patients affected with T2D and 534 non-diabetic individuals were recruited at the University Hospital in Leipzig, Germany. The group of patients with T2D included 377 males and 308 females (mean age $66.2 \pm 0.3$ years, mean BMI $28.9 \pm 0.1$ ). In all non-diabetic patients an oral glucose tolerance test (OGTT) and fasting plasma insulin measurement were performed. The OGTT was performed according to the criteria of the 
American Diabetes Association (ADA) (Genuth et al. 2003). The test was carried out after an overnight fast with $75 \mathrm{~g}$ standardized glucose solution (Glucodex Solution $75 \mathrm{~g}$, Merieux Ltd, Canada). Venous blood samples were taken at 0,60 and $120 \mathrm{~min}$ for measurements of plasma glucose concentrations. A total of 461 (121 males and 340 females; mean age $47.8 \pm 0.5$ years, mean BMI $28.6 \pm 0.2$ ) out of 534 subjects had normal glucose tolerance (NGT). All 461 subjects were included in the control cohort and had no family history of T2D. All studies were approved by the Ethics Committee of the University of Leipzig, and all subjects gave written informed consent before taking part in the study.

Genotyping of the R1467H was done using the TaqMan allelic discrimination assay (Applied Biosystems, Inc.).

We performed two case-control studies using 461 healthy individuals versus both $685 \mathrm{~T} 2 \mathrm{D}$ cases as well as a combined group of cases including 685 subjects affected with T2D and 73 individuals with IGT (T2D/IGT, $N=758)$. The $\mathrm{H} 1467$ variant was significantly more frequent in T2D cases $(50.5 \%)$ or the T2D/IGT group (50.9\%) than in healthy controls $(46.0 \%)(P=0.004$ and $P=0.001$, respectively, in an additive model under logistic regression analysis after adjustments for age, sex and BMI; Table 1). Thus, our data replicate the initial findings in Pima Indians, suggesting the $\mathrm{H}$ allele as the diabetes risk allele (Ma et al. 2007). Considering the known Swiss-German ancestry of the Amish, it may be surprising that our present data in German Caucasians confirm the Pima findings rather than those reported in the Amish (Fu et al. 2007). However, because of the relatively small sample size and only a nominally significant association of the common $\mathrm{R}$ allele with T2D in the Amish $(P=0.04)$, we believe that our present findings consistent with those in Pima Indians strongly suggest the role of the variant in the pathophysiology of T2D. Nevertheless, we are aware that additional investigations in independent populations as well as functional studies will be inevitable to clarify the influence of the ARHGEF11 variant on T2D. Also, it cannot be precluded that the $\mathrm{R} 1467 \mathrm{H}$ may not be the functional variant, but rather is in linkage disequilibrium with a true functional variant.

Consistent with our finding on T2D, carriers of the diabetes risk allele tended to have a higher mean 2-h blood glucose in the OGTT. Subjects carrying the homozygous major $\mathrm{R}$ allele $(N=153)$ have lower mean 2-h blood glucose levels $(5.94 \pm 0.12 \mathrm{mmol} / \mathrm{l})$ compared to heterozygous individuals $(N=259 ; 6.21 \pm 0.10 \mathrm{mmol} / \mathrm{l})$ or carriers of two copies of the derived $\mathrm{H}$ allele $(N=122$; $6.26 \pm 0.15 \mathrm{mmol} / \mathrm{l})(P=0.08$ in generalized linear model analyses after adjustments for age, sex and BMI).

In conclusion, our study replicated associations of the ARHGEF11 polymorphism with increased risk of T2D or

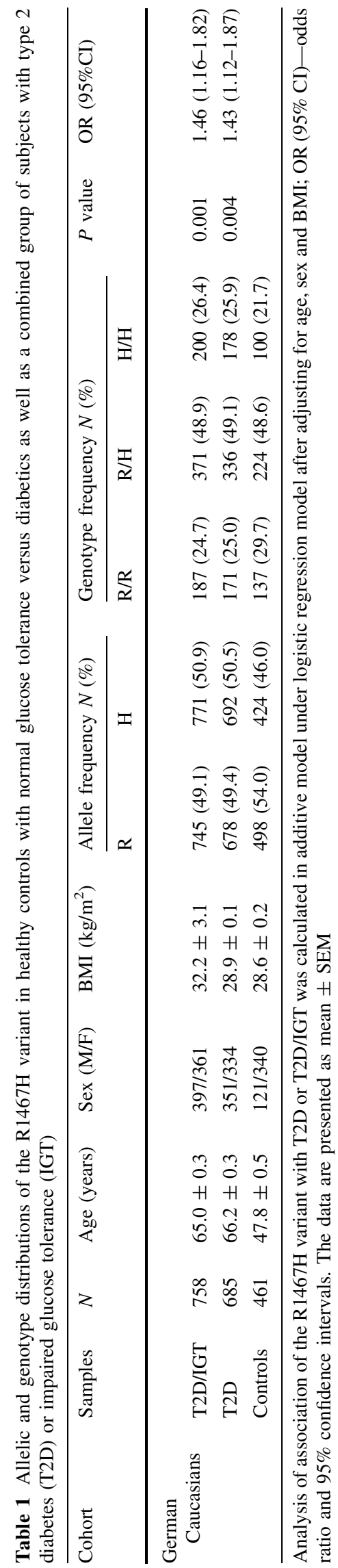


T2D/IGT in a German Caucasian population and thus supports previous data implicating a potential role of $A R$ HGEF 11 in the aetiology of T2D and IGT. Further replication studies in different ethnic populations are necessary to confirm the effect of the R1467H variant on T2D.

Acknowledgments We thank all those who participated in the studies. We appreciate the help of the nurses and physicians who performed the clinical examinations and data collection. This work was supported by grants from the Medical Faculty at the University of Leipzig (formel.1 to Y.B.), from the Deutsche Forschungsgemeinschaft (DFG) KFO 152: "Atherobesity" (projects BL 833/1-1 (M.B.), Stu192/6-1 (M.S.) and from the Interdisciplinary Centre of Clinical Research (IZKF) Leipzig at the Faculty of Medicine of the University of Leipzig (project N06 to D.S. and P.K. and project B24 to M.B., B27 to M.S.).

\section{References}

Fu M, Sabra MM, Damcott C, Pollin TI, Ma L, Ott S, Shelton JC, Shi X, Reinhart L, O'Connell J, Mitchell BD, Baier LJ, Shuldiner AR (2007) Evidence that Rho guanine nucleotide exchange factor 11 (ARHGEF11) on 1q21 is a type 2 diabetes susceptibility gene in the Old Order Amish. Diabetes 56:1363-1368

Genuth S, Alberti KG, Bennett P, Buse J, Defronzo R, Kahn R, Kitzmiller J, Knowler WC, Lebovitz H, Lernmark A, Nathan D, Palmer J, Rizza R, Saudek C, Shaw J, Steffes M, Stern M, Tuomilehto J, Zimmet P (2003) Follow-up report on the diagnosis of diabetes mellitus. Diabetes Care 26:3160-3167

Larsen L, Storling J, Darville M, Eizirik DL, Bonny C, Billestrup N, Mandrup-Poulsen T (2005) Extracellular signal-regulated kinase is essential for interleukin-1-induced and nuclear factor kappaBmediated gene expression in insulin-producing INS-1E cells. Diabetologia 48:2582-2590

Ma L, Hanson RL, Que LN, Cali AM, Fu M, Mack JL, Infante AM, Kobes S, Bogardus C, Shuldiner AR, Baier LJ (2007) Variants in ARHGEF11, a candidate gene for the linkage to type 2 diabetes on chromosome 1q, are nominally associated with insulin resistance and type 2 diabetes in Pima Indians. Diabetes 56:1454-1459

McCarthy MI (2003) Growing evidence for diabetes susceptibility genes from genome scan data. Curr Diab Rep 3:159-167

Nevins AK, Thurmond DC (2005) A direct interaction between Cdc42 and vesicle-associated membrane protein 2 regulates SNARE-dependent insulin exocytosis. J Biol Chem 280:19441952 